\title{
IFSs consisting of generalized convex contractions
}

\section{Flavian Georgescu}

\begin{abstract}
In this paper we introduce the concept of iterated function system consisting of generalized convex contractions. More precisely, given $n \in \mathbb{N}^{*}$, an iterated function system consisting of generalized convex contractions on a complete metric space $(X, d)$ is given by a finite family of continuous functions $\left(f_{i}\right)_{i \in I}, f_{i}: X \rightarrow X$, having the property that for every $\omega \in \Lambda_{n}(I)$ there exists a family of positive numbers $\left(a_{\omega, v}\right)_{v \in V_{n}(I)}$ such that:

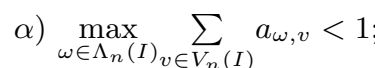

B) $d\left(f_{\omega}(x), f_{\omega}(y)\right) \leq \sum_{v \in V_{n}(I)} a_{\omega, v} d\left(f_{v}(x), f_{v}(y)\right)$ for all $\omega \in \Lambda_{n}(I)$, $x, y \in X$. Here $\Lambda_{n}(I)$ represents the family of words with $n$ letters from $I, V_{n}(I)$ designates the family of words having at most $n-1$ letters from $I$, while, if $\omega=\omega_{1} \omega_{2} \ldots \omega_{p}$, by $f_{\omega}$ we mean $f_{\omega_{1}} \circ f_{\omega_{2}} \circ \ldots \circ f_{\omega_{p}}$. Denoting such a system by $\mathcal{S}=\left((X, d), n,\left(f_{i}\right)_{i \in I}\right)$, one can consider the function $F_{\mathcal{S}}: \mathcal{K}(X) \rightarrow \mathcal{K}(X)$ described by $F_{\mathcal{S}}(B)=\cup_{i \in I} f_{i}(B)$, for all $B \in \mathcal{K}(X)$, where $\mathcal{K}(X)$ means the set of non-empty compact subsets of $X$. Our main result states that $F_{\mathcal{S}}$ is a Picard operator for every iterated function system consisting of generalized convex contractions $\mathcal{S}$.
\end{abstract}

\section{Introduction}

As the contraction condition from Banach-Caccioppoli-Picard principle is very strong, V. Istrăţescu introduced and studied the convex contraction condition (see [9], [10] and [11]) in order to provide contraction-type conditions which do

Key Words: generalized convex contractions, fixed points, iterated function systems consisting of generalized convex contractions.

2010 Mathematics Subject Classification: Primary 28A80; Secondary 54H20.

Received: 12.03.2016.

Accepted: 28.04.2016. 
not imply the contraction condition but for which the existence and uniqueness of the fixed point are assured. More precisely we have the following:

Definition 1.1. A continuous function $f: X \rightarrow X$, where $(X, d)$ is a complete metric space, is called convex contraction if there exist $a, b \in(0,1)$ such that $a+b<1$ and $\left.d\left(f^{[2]}(x), f^{[2]}(y)\right) \leq a d(f(x)), f(y)\right)+b d(x, y)$ for every $x, y \in X$.

Istrăţescu proved that any convex contraction is a Picard operator. In addition he presented a convex contraction which is not contraction and introduced the following generalization of the concept of convex contraction that was also studied by S. András (see [2] and [3]):

Definition 1.2. Given a complete metric space $(X, d)$, a continuous function $f: X \rightarrow X$ is called a generalized convex contraction provided that there exist $n \in \mathbb{N}^{*}$ and $\alpha_{0}, \alpha_{1}, \ldots, \alpha_{n-1} \geq 0$ such that $\sum_{i=0}^{n-1} \alpha_{i}<1$ and $d\left(f^{[n]}(x), f^{[n]}(y)\right) \leq$ $\sum_{k=0}^{n-1} \alpha_{k} d\left(f^{[k]}(x), f^{[k]}(y)\right)$ for all $x, y \in X$, where by $f^{[k]}$ we mean the composition of $f$ by itself $k$ times.

They proved that each generalized convex contraction is a Picard operator. For other generalizations of Istrățescu's result see [1], [12], [14], [16], [20] and [29].

For an iterated function system consisting of a finite family of contractions $\left(f_{k}\right)_{k \in\{1,2, \ldots, n\}}, f_{k}: X \rightarrow X$, where $(X, d)$ is a complete metric space, there exists a unique non-empty compact subset $A$ (called the attractor of the system) of $X$ such that $A=\bigcup_{k=1}^{n} f_{k}(A)$. This procedure gives almost all fractals and consequently several authors extended the notion of iterated function system (see [4], [5], [6], [7], [8], [13], [15], [17], [18], [19], [21], [22], [23], [25], [26], [27], [28], [30], [31], [32], [33] and [34] and the references therein). Along these lines of research R. Miculescu and A. Mihail (see [24]) introduced the concept of iterated function system consisting of convex contractions and proved the existence and uniqueness of the attractor of such a system obtaining in this way another generalization of the above mentioned Istrățescu's result.

In this paper, combining these two directions of generalization of Istrățescu's theorem, we study iterated function systems consisting of generalized convex contractions.

\section{Preliminaries}

By $f^{[n]}$ we mean the composition of the function $f: X \rightarrow X$ by itself $n$ times. 
For a family of functions $\left(f_{i}\right)_{i \in I}$, where $f_{i}: X \rightarrow X, \alpha_{1}, \alpha_{2}, \ldots, \alpha_{n} \in I$ and $Y \subseteq X$, we use the following notations:

$-f_{\alpha_{1}} \circ f_{\alpha_{2}} \circ \ldots \circ f_{\alpha_{n}} \stackrel{\text { not }}{=} f_{\alpha_{1} \alpha_{2} \ldots \alpha_{n}}$

$-f_{\alpha_{1} \alpha_{2} \ldots \alpha_{n}}(Y) \stackrel{\text { not }}{=} Y_{\alpha_{1} \alpha_{2} \ldots \alpha_{n}}$.

For a metric space $(X, d)$, by $\mathcal{K}(X)$ we mean the set of non-empty compact subsets of $X$.

$B^{A}$ represents the set of functions from $A$ to $B$.

For a set $I$, we use the following notations:

$-I^{\mathbb{N}^{*}} \stackrel{\text { not }}{=} \Lambda(I)$; hence the elements of $\Lambda(I)$ can be written as infinite words $\omega=\omega_{1} \omega_{2} \ldots \omega_{n} \ldots$ with letters from $I$

- $I^{\{1,2, \ldots, n\}} \stackrel{\text { not }}{=} \Lambda_{n}(I)$; hence the elements of $\Lambda_{n}(I)$ can be written as words $\omega=\omega_{1} \omega_{2} \ldots \omega_{n}$ with $n$ letters from $I$

$-\Lambda_{0}(I) \cup \Lambda_{1}(I) \cup \ldots \cup \Lambda_{n-1}(I) \stackrel{\text { not }}{=} V_{n}(I)$, where $\Lambda_{0}(I)=\{\lambda\}$ is the set consisting on the empty word; hence $V_{n}(I)$ is the set of all words having at most $n-1$ letters from $I$ from $I$.

- $\cup_{n \in \mathbb{N}} \Lambda_{n}(I) \stackrel{\text { not }}{=} \Lambda^{*}(I)$; hence $\Lambda^{*}(I)$ is the set of all finite words with letters

For a function $f: X \rightarrow X$, by $f_{\lambda}$ we mean $I d_{X}$. For $\alpha \in \Lambda(I) \cup \Lambda_{n}(I)$ and $m \leq n$, we use the following notation: $\alpha_{1} \alpha_{2} \ldots \alpha_{m} \stackrel{\text { not }}{=}[\alpha]_{m}$.

By $\alpha \beta$ we understand the concatenation of the words $\alpha \in \Lambda^{*}(I)$ and $\beta \in$ $\Lambda(I) \cup \Lambda^{*}(I)$.

Definition 2.1. For a metric space $(X, d)$, we consider the Hausdorff-Pompeiu metric $h: \mathcal{K}(X) \times \mathcal{K}(X) \rightarrow[0,+\infty)$ described by

$$
h(A, B)=\max \left\{\sup _{x \in A}\left(\inf _{y \in B} d(x, y)\right), \sup _{x \in B}\left(\inf _{y \in A} d(x, y)\right)\right\}
$$

for every $A, B \in \mathcal{K}(X)$.

Proposition 2.2. (see Proposition 2.1 from [26]) For a metric space $(X, d)$, we have

$$
h\left(\cup_{i \in I} H_{i}, \cup_{i \in I} K_{i}\right) \leq \sup _{i \in I} h\left(H_{i}, K_{i}\right),
$$

for every $\left(H_{i}\right)_{i \in I}$ and $\left(K_{i}\right)_{i \in I}$ finite families of elements from $\mathcal{K}(X)$.

Proposition 2.3. (see Theorem 2.1 from [26]) If the metric space $(X, d)$ is complete, then $(\mathcal{K}(X), h)$ is a complete metric space. 
Definition 2.4. For a metric space $(X, d)$, we consider the function $\delta$ : $\mathcal{K}(X) \times \mathcal{K}(X) \rightarrow[0,+\infty)$ defined by

$$
\delta(A, B)=\sup _{x \in A, y \in B} d(x, y)
$$

for all $A, B \in \mathcal{K}(X)$.

Remark 2.5. For all $A, B \in \mathcal{K}(X)$ we have

$$
h(A, B) \leq \delta(A, B) .
$$

Definition 2.6. A function $f: X \rightarrow X$, where $(X, d)$ is a metric space, is called Picard operator if there exists a unique $x^{*} \in X$ such that $f\left(x^{*}\right)=x^{*}$ and $\lim _{k \rightarrow \infty} f^{[k]}(x)=x^{*}$ for every $x \in X$.

\section{The main result}

Definition 3.1. Given $n \in \mathbb{N}^{*}$, an iterated function system consisting of generalized convex contractions on a complete metric space $(X, d)$ is given by a finite family of continuous functions $\left(f_{i}\right)_{i \in I}, f_{i}: X \rightarrow X$, such that for every $\omega \in \Lambda_{n}(I)$ there exists a family of positive numbers $\left(a_{\omega, v}\right)_{v \in V_{n}(I)}$ such that:

$\alpha)$

$$
\max _{\omega \in \Lambda_{n}(I)} \sum_{v \in V_{n}(I)} a_{\omega, v}<1
$$

$$
d\left(f_{\omega}(x), f_{\omega}(y)\right) \leq \sum_{v \in V_{n}(I)} a_{\omega, v} d\left(f_{v}(x), f_{v}(y)\right),
$$

for all $\omega \in \Lambda_{n}(I), x, y \in X$.

We denote such a system by

$$
\mathcal{S}=\left((X, d), n,\left(f_{i}\right)_{i \in I}\right) .
$$

One can consider the function $F_{\mathcal{\delta}}: \mathcal{K}(X) \rightarrow \mathcal{K}(X)$ described by

$$
F_{\mathcal{S}}(B)=\bigcup_{i \in I} f_{i}(B)
$$

for all $B \in \mathcal{K}(X)$. 
Remark 3.2. If the set I has one element, $\left((X, d), n,\left(f_{i}\right)_{i \in I}\right)$ is nothing else but the notion of generalized convex contraction. Note also that the notion of iterated function system consisting of convex contractions from [24] is a particular case of the one of iterated function system consisting of generalized convex contractions (just take $n=2$ ).

Theorem 3.3. $F_{\mathcal{S}}$ is a Picard operator, for every iterated function system consisting of generalized convex contractions $\mathcal{S}$.

Proof. In the sequel we shall use the following notations:

$-\mathcal{S}=\left((X, d), n,\left(f_{i}\right)_{i \in I}\right)$

$-\sum_{v \in V_{n}(I)} a_{\omega, v} \stackrel{\text { not }}{=} d_{\omega}$

$-\max _{\omega \in \Lambda_{n}(I)} d_{\omega} \stackrel{\text { not }}{=} d<1$

$-y_{k}(Y, Z)=\max \left\{x_{k-n+1}(Y, Z), x_{k-n+2}(Y, Z), \ldots, x_{k}(Y, Z)\right\}$, where $x_{k}(Y, Z)=\sup _{\omega \in \Lambda_{k}(I)} \delta\left(f_{\omega}(Y), f_{\omega}(Z)\right), Y, Z \in \mathcal{K}(X), k \in \mathbb{N}^{*}, k \geq n-1$ and $Y, Z \in \mathcal{K}(X)$.

When no confusion is possible, we denote $y_{k}(Y, Z)$ by $y_{k}$ and $x_{k}(Y, Z)$ by $x_{k}$.

Claim 3.4. The sequence $\left(y_{k+n}(Y, Z)\right)_{k \in \mathbb{N}^{*}}$ is decreasing for all $Y, Z \in \mathcal{K}(X)$.

Justification of Claim 3.4. Given $u \in \Lambda_{k+n}(I)$ there exist $\omega \in \Lambda_{n}(I)$ and $q \in \Lambda_{k}(I)$ such that $u=\omega q$ and therefore we have

$$
\begin{gathered}
d\left(f_{u}(y), f_{u}(z)\right)=d\left(f_{\omega q}(y), f_{\omega q}(z)\right) \leq \\
\leq \sum_{v \in V_{n}} a_{\omega, v} d\left(f_{v q}(x), f_{v q}(y)\right) \leq a_{\omega} x_{k}+\sum_{v \in \Lambda_{1}(I)} a_{\omega, v} x_{k+1}+\ldots+\sum_{v \in \Lambda_{n-1}(I)} a_{\omega, v} x_{k+n-1} \leq \\
\leq \max \left\{x_{k}, x_{k+1}, \ldots, x_{k+n-1}\right\}\left(a_{\omega}+\sum_{v \in \Lambda_{1}(I)} a_{\omega, v}+\ldots+\sum_{v \in \Lambda_{n-1}(I)} a_{\omega, v}\right) \leq \\
\leq y_{k+n-1} \sum_{v \in V_{n}(I)} a_{\omega, v}=d_{\omega} y_{k+n-1} \leq d y_{k+n-1},
\end{gathered}
$$

for all $y \in Y$ and $z \in Z$, so, by passing to supremum over $y \in Y$ and $z \in Z$, we deduce that $\delta\left(f_{u}(Y), f_{u}(Z)\right) \leq d y_{k+n-1}$ for all $k \in \mathbb{N}^{*}$. By passing to supremum over $u \in \Lambda_{k+n}(I)$, we get that

$$
x_{k+n}=\sup _{\omega \in \Lambda_{k+n}(I)} \delta\left(f_{\omega}(Y), f_{\omega}(Z)\right) \leq d y_{k+n-1}<y_{k+n-1},
$$


for all $k \in \mathbb{N}^{*}$. As

$$
x_{k+1}, x_{k+2}, \ldots, x_{k+n-1} \leq \max \left\{x_{k}, x_{k+1}, \ldots, x_{k+n-1}\right\}=y_{k+n-1},
$$

from $(*)$ and $(* *)$, we infer that $y_{k+n}=\max \left\{x_{k+1}, x_{k+2}, \ldots, x_{k+n}\right\} \leq y_{k+n-1}$ for all $k \in \mathbb{N}^{*}$ and the justification of Claim 3.4 is done.

Claim 3.5. $\lim _{k \rightarrow \infty} x_{k}(Y, Z)=0$ for all $Y, Z \in \mathcal{K}(X)$.

Justification of Claim 3.5 Based on (*) from the proof of Claim 3.4, we have

$$
\begin{gathered}
y_{k+2 n-1}=\max \left\{x_{k+n}, x_{k+1+n}, \ldots, x_{k+2 n-1}\right\} \leq \\
\leq \max \left\{d y_{k+n-1}, d y_{k+n}, \ldots, d y_{k+2 n-2}\right\},
\end{gathered}
$$

so, taking into account Claim 3.4, we get that $y_{k+2 n-1} \leq d y_{k+n-1}$ for all $k \in$ $\mathbb{N}^{*}$. Consequently $y_{n i} \leq d^{i-1} y_{n}, y_{n i+1} \leq d^{i-1} y_{n+1} \leq d^{i-1} y_{n}, \ldots, y_{n i+n-1} \leq$ $d^{i-1} y_{2 n-1} \leq d^{i-1} y_{n}$ for all $i \in \mathbb{N}^{*}$, hence the series $\sum_{k=n}^{\infty} y_{k}$ is convergent. Using (*) from the proof of Claim 3.4 and the comparison test, we conclude that the series $\sum_{k=n+1}^{\infty} x_{k}$ is convergent and, consequently, $\lim _{k \rightarrow \infty} x_{k}=0$. The justification of Claim 3.5 is done.

Claim 3.6. For every $Y \in \mathcal{K}(X)$, the sequence $\left(F_{\mathcal{S}}^{[k]}(Y)\right)_{k \in \mathbb{N}^{*}}$ is convergent.

Justification of Claim 3.6 Since

$$
\begin{gathered}
h\left(F_{\mathcal{S}}^{[k]}(Y), F_{\mathcal{S}}^{[k]}(Z)\right)=h\left(\underset{\omega \in \Lambda_{k}(I)}{\cup} f_{\omega}(Y), \underset{\omega \in \Lambda_{k}(I)}{\cup} f_{\omega}(Z)\right) \stackrel{\text { Proposition } 2.2}{\leq} \\
\leq \sup _{\omega \in \Lambda_{k}(I)} h\left(f_{\omega}(Y), f_{\omega}(Z)\right) \stackrel{\text { Remark } 2.5}{\leq} x_{k},
\end{gathered}
$$

for every $Y, Z \in \mathcal{K}(X), k \in \mathbb{N}^{*}$, we deduce that

$$
\lim _{k \rightarrow \infty} h\left(F_{\mathcal{S}}^{[k]}(Y), F_{\mathcal{S}}^{[k]}(Z)\right)=0 .
$$

Choosing $Z=F_{\delta}(Y)$, from $(*)$, the convergence of the series $\sum_{k} x_{k}$ and the comparison test, we infer that the series $\sum_{k \in \mathbb{N}^{*}} h\left(F_{\mathcal{S}}^{[k+1]}(Y), F_{\mathcal{S}}^{[k]}(Y)\right)$ is convergent for all $Y \in \mathcal{K}(X)$. Therefore $\left(F_{S}^{[k]}(Y)\right)_{k \in \mathbb{N}^{*}}$ is a Cauchy sequence and, because $(\mathcal{K}(X), h)$ is complete (see Proposition 2.3), it is convergent. The justification of Claim 3.6 is done. 
Claim 3.6 assures us that if $Y, Z \in \mathcal{K}(X)$, then there exist $A_{Y}, A_{Z} \in \mathcal{K}(X)$ such that

$$
\lim _{k \rightarrow \infty} h\left(F_{\mathcal{S}}^{[k]}(Y), A_{Y}\right)=0 \text { and } \lim _{k \rightarrow \infty} h\left(F_{\mathcal{S}}^{[k]}(Z), A_{Z}\right)=0 .
$$

Since $h\left(A_{Y}, A_{Z}\right) \leq h\left(A_{Y}, F_{\mathcal{S}}^{[k]}(Y)\right)+h\left(F_{\mathcal{S}}^{[k]}(Y), F_{\mathcal{S}}^{[k]}(Z)\right)+h\left(F_{\mathcal{S}}^{[k]}(Z), A_{Z}\right)$ for all $k \in \mathbb{N}^{*}$, from (1) and (2) we obtain that $A_{Y}=A_{Z} \stackrel{\text { def }}{=} A$ for every $Y, Z \in \mathcal{K}(X)$. Hence $\lim _{k \rightarrow \infty} h\left(F_{\mathcal{S}}^{[k]}(B), A\right)=0$ for every $B \in \mathcal{K}(X)$. The remaining part of the proof goes as in Theorem 3.2 from [24] and, consequently, we just mark the main steps:

a) For each $\omega \in \Lambda(I)$ there exists $a_{\omega} \in X$ such that

$$
\lim _{k \rightarrow \infty} \sup _{\omega \in \Lambda(I)} h\left(f_{[\omega]_{k}}(B),\left\{a_{\omega}\right\}\right)=0,
$$

for every $B \in \mathcal{K}(X)$.

b)

$$
A=\overline{\left\{a_{\omega} \mid \omega \in \Lambda(I)\right\}}
$$

c) The implication

$$
\lim _{k \rightarrow \infty} h\left(Y_{k}, Y\right)=0 \Rightarrow \lim _{k \rightarrow \infty} h\left(F_{\mathcal{S}}\left(Y_{k}\right), F_{\mathcal{S}}(Y)\right)=0
$$

is true for every $\left(Y_{k}\right)_{k \in \mathbb{N}} \subseteq \mathcal{K}(X)$ and $Y \in \mathcal{K}(X)$.

d) $A$ is the unique fixed point of $F_{\mathcal{S}}$.

Conclusions. In this paper we consider a special type of iterated function systems, namely those consisting of generalized contractions. We proved that such a system has a unique attractor, obtaining in this way a generalization of a fixed point theorem concerning generalized convex contractions which is due to V. Istrăţescu. In a future paper we intend to study iterated function systems consisting of generalized convex contractions in the more general setting of b-metric spaces.

Acknowledgement. The author thanks to the referees for their valuable remarks and comments which lead to the paper's improvement.

\section{References}

[1] M.A. Alghamdia, S.H. Alnafeia, S. Radenović, N. Shahzad, Fixed point theorems for convex contraction mappings on cone metric spaces, Math. Comput. Modelling, 54 (2011), 2020-2026. 
[2] Sz. András, Fiber Picard operators and convex contractions, Fixed Point Theory, 4 (2003), 121-129.

[3] Sz. András, Ecuaţii integrale Fredholm-Volterra, Editura Didactică şi Pedagogică, Bucureşti, 2005.

[4] J. Andres and M. Rypka, Multivalued fractals and hyperfractals, Internat. J. Bifur. Chaos Appl. Sci. Engrg., 22 (2012), DOI 10.1142/S02181127412500095.

[5] M. Barnsley, K. Leśniak and M. Rypka, Chaos game for IFSs on topological spaces, J. Math. Anal. Appl. 435, (2016), 1458-1466.

[6] M. Boriceanu, M. Bota and A. Petruşel, Multivalued fractals in b-metric spaces, Cent. Eur. J. Math., 8 (2010), 367-377.

[7] C. Chifu and A. Petruşel, Multivalued fractals and generalized multivalued contractions, Chaos Solitons Fractals, 36 (2008), 203-210.

[8] I. Chiţescu, H. Georgescu, R. Miculescu, Approximation of infinite dimensional fractals generated by integral equations, J. Comput. Appl. Math., $234(2-10)$ 1417-1425.

[9] V. Istrăţescu, Some fixed point theorems for convex contraction mappings and convex nonexpansive mappings (I), Libertas Math., 1 (1981), 151164.

[10] V. Istrățescu, Some fixed point theorems for convex contraction mappings and mappings with convex diminishing diameters - I, Annali di Mat. Pura Appl., 130 (1982), 89-104.

[11] V. Istrățescu, Some fixed point theorems for convex contraction mappings and mappings with convex diminishing diameters - II, Annali di Mat. Pura Appl., 134 (1983), 327-362.

[12] V. Ghorbanian, S. Rezapour and N. Shahzad, Some ordered fixed point results and the property (P), Comput. Math. Appl., 63 (2012), 1361-1368.

[13] G. Gwóźdź-Łukowska and J. Jachymski, IFS on a metric space with a graph structure and extensions of the Kelisky-Rivlin theorem, J. Math. Anal. Appl., 356 (2009), 453-463.

[14] N. Hussain, M. A. Kutbi, S. Khaleghizadeh and P. Salimi, Discussions on recent results for $\alpha-\Psi$-contractive mappings, Abstr. Appl. Anal., vol. 2014, Article ID 456482, 13 pages, 2014. 
[15] M. Klimek and M. Kosek, Generalized iterated function systems, multifunctions and Cantor sets, Ann. Polon. Math., 96 (2009), 25-41.

[16] A. Latif, W. Sintunavarat and A. Ninsri, Approximate fixed point theorems for partial generalized convex contraction mappings in $\alpha$-complete metric spaces, Taiwanese J. Math., 19 (2015), 315-333.

[17] K. Leśniak, Infinite iterated function systems: a multivalued approach, Bull. Pol. Acad. Sci. Math., 52 (2004), 1-8.

[18] E. Llorens-Fuster, A. Petruşel and J.-C. Yao, Iterated function systems and well posedness, Chaos Solitons Fractals, 41 (2009), 1561-1568.

[19] L. Máté, The Hutchinson-Barnsley theory for certain noncontraction mappings, Period. Math. Hungar., 27 (1993), 21-33.

[20] M. A. Miandaragh, M. Postolache and S. Rezapour, Approximate fixed points of generalized convex contractions, Fixed Point Theory Appl., vol. 2013, article 255, 2013.

[21] R. Miculescu, Generalized iterated function systems with place dependent probabilities, Acta Appl. Math., 130 (2014),135-150.

[22] R. Miculescu and A. Mihail, On a question of A. Kameyama concerning self-similar metrics, J. Math. Anal. Appl., 422 (2015), 265-271.

[23] R. Miculescu and A. Mihail, Reich-type iterated function systems, J. Fixed Point Theory Appl., 18 (2016), 285-296.

[24] R. Miculescu and A. Mihail, A generalization of Istrăţescu's fixed point theorem for convex contractions, in print, Fixed Point Theory.

[25] A. Mihail and R. Miculescu, Applications of fixed point theorems in the theory of generalized IFS, Fixed Point Theory Appl., 2008, Art. ID 312876, $11 \mathrm{pp}$.

[26] A. Mihail and R. Miculescu, The shift space for an infinite iterated function system, Math. Rep. (Bucur.), 61 (2009), 21-31.

[27] A. Mihail and R. Miculescu, Generalized IFSs on noncompact spaces, Fixed Point Theory Appl., 2010, Art. ID 584215, 15 pp.

[28] A. Mihail, A topological version of iterated function systems, An. Ştiinţ . Univ. Al. I. Cuza, Iaşi, (S.N.), Matematica, 58 (2012) 105-120.

[29] V. Mureşan and A. Mureşan, On the theory of fixed point theorems for convex contraction mappings, Carpathian J. Math., 31 (2015), 365-371. 
[30] A. Petruşel, Iterated function system of locally contractive operators, Rev. Anal. Numér. Théor. Approx., 33 (2004), 215-219.

[31] N. A. Secelean, Iterated function systems consisting of F-contractions, Fixed Point Theory Appl., 2013, 2013:277.

[32] N. A. Secelean, Generalized iterated function systems on the space $l^{\infty}(X)$, J. Math. Anal. Appl., 410 (2014), 847-458.

[33] F. Strobin and J. Swaczyna, On a certain generalization of the iterated function systems, Bull. Australian Math. Soc., 87 (2013), 37-54.

[34] F. Strobin, Attractors of generalized IFSs that are not attractors of IFSs, J. Math. Anal. Appl., 422 (2015), 99-108.

Flavian GEORGESCU,

Faculty of Mathematics and Computer Science,

University of Piteşti, Romania,

Târgul din Vale 1, 110040, Piteşti, Argeş, Romania.

Email: georgescu.flavian@ichb.ro 